\section{AB0507 BELIMUMAB IN PATIENTS WITH SYSTEMIC LUPUS ERYTHEMATOSUS: OUTCOMES IN ROUTINE CLINICAL PRACTICE}

C. Moriano $^{1}$, M. Garijo ${ }^{1}$, C. Iñiguez ${ }^{1}$, E. Díez ${ }^{1}$, C. Díez ${ }^{2}$, L. Gómez-Lechón ${ }^{3}$, J. Martín ${ }^{4}$, M. Corteguera ${ }^{5}$, O. Martínez ${ }^{3}$, J. Cabezas ${ }^{6}$, T. Pérez ${ }^{1} .{ }^{1}$ Hospital León, León; ${ }^{2}$ Hospital El Bierzo, Ponferrada; ${ }^{3}$ Hospital Salamanca, Salamanca; ${ }^{4}$ Hospital Río Hortega, Valladolid; ${ }^{5}$ Hospital Ávila, Ávila; ${ }^{6}$ Hospital Zamora, Zamora, Spain

Background: Systemic lupus erythematosus (SLE) is a systemic autoimmune disease with a wide range of clinical manifestations. The persistence of activity of the disease and the prolonged use of corticosteroids are amongst the main predictors of accumulated organ damage in patients with SLE. Therefore, we need new strategies to induce long-lasting remission and minimise the adverse effects of standard medications.

Objectives: to assess the effectiveness and safety of Belimumab in actual clinical practice during the first year of treatment in patients with SLE.

Methods: retrospective observational study of patients treated with Belimumab. This study involves 6 hospitals in Castilla y León (Spain). It collects clinical and analytical data before the start of Belimumab, and 6 and 12 months after the initiation of the therapy. The data collected include the daily dose of prednisone received at different time points and the activity of the disease was classified as mild, moderate and severe based on SELENA-SLEDAI index. A reduction from baseline of 3 points on the SELENA-SLEDAI indicates meaningful symptomatic improvement.

Results: Among the twenty-five patients were included, with a mean age of 43.7 +12.2 years, $72 \%$ were female and $96 \%$ caucasian. The average time from diagnosis of SLE to Belimumab infusion was $9.5+7.1$ years; $32 \%$ were diagnosed with SLE $<5$ years ago. Up to $56 \%$ of the patients had moderate disease activity and only $8 \%$ patients had very high activity of the disease. All but one of the patients had previously received an immunosuppressant (azathioprine in 50\% of the cases) and $16 \%$ of the sample had not responded to rituximab. The most frequent reasons for initiating Belimumab were an ineffective previous treatment regimen $(60 \%)$, the intend to decrease steroid use $(56 \%)$, and worsening patient condition $(52 \%)$; whereas the most frequent manifestation of SLE in these patients were arthritis $(44 \%)$, followed by mucocutaneous $(20 \%)$ and immunologic findings (20\%). As for immunologic markers of activity, DNA was elevated by $40 \%$ and $68 \%$ of the patients showed hypocomplementemia, that dropped to $24 \%$ in both cases at 12 months. The mean score of the SELENA-SLEDAl index decreased from 9.5 to 6 in the first 6 months and to 5.2 in the 12 months of follow-up. A reduction in the initial mean steroid dose was also observed from $20 \mathrm{mg} /$ day to $8.9 \mathrm{mg} /$ day and $6.4 \mathrm{mg} /$ day at 6 and 12 months respectively. Hence, the dose of steroid was reduced by $68 \%$ during the first year $(p=0,06)$. This reduction was clinically significant, but it did not reach statistical significance in our study. Two patients $(8 \%)$ had discontinued Belimumab within the first 6 months of therapy (1 pregnancy and 1 worsening proteinuria); $34.7 \%$ had discontinued it within the first 12 months, mostly due to ineffectiveness $(62.5 \%)$. Two patients with severe renal involvement (>1 gr proteinuria/24 hour) were treated with Belimumab, allowing to reduce the steroids to a low dosis ( $<7.5 \mathrm{mg} /$ day) at 12 months.

Conclusions: Belimumab appears to be effective, reducing disease activity as measured by the SELENA-SLEDAI Index and, also, the mean dose of steroids needed, mainly after 12 months of treatment. No significant adverse effects were reported as previous studies have shown.

Disclosure of Interest: None declared

DOI: 10.1136/annrheumdis-2018-eular.7530

\section{AB0508 FIXED, LOW-DOSE HYDROXICHLOROQUINE VERSUS WEIGHT-ADJUSTED DOSE IN SYSTEMIC LUPUS ERYTHEMATOSUS PATIENTS WITH LOW ACTIVITY}

J.L. Callejas-Rubio ${ }^{1}$, D. Sanchez-Cano ${ }^{1}$, R. Ríos-Fernández ${ }^{1}$, M. MorenoHigueras $^{2}$, G. Fatoul del Pino ${ }^{1}$, S. Velasco-Fuentes ${ }^{1}$, M. Trigo-Rodríguez ${ }^{1}$, N. FaroMínguez ${ }^{1} .{ }^{1}$ Unidad de Enfermedades Autoinmunes Sistémicas; ${ }^{2}$ Servicio de Medicina Interna, Hospital Campus de la Salud, Granada, Spain

Background: Optimal hydroxichloroquine (HCQ) dose to reduce ocular toxicity risk is $5 \mathrm{mg} / \mathrm{kg}$ or actual weight daily, according to new recommendations. In systemic lupus erythematosus patients (SLE) with low or no disease activity, fixed low-dose HCQ might be equally effective in relapse prevention weight-adjusted doses, thus resulting in lower cumulative dose.

Objectives: Our aim was to compare a fixed dose of HCQ $200 \mathrm{mg}$ daily with a weight-adjusted dose $(5 \mathrm{mg} / \mathrm{kg}$ of actual weight daily) in SLE patients with low activity.

Methods: SLE activity was assessed using the SLE Disease Activity Index (SLEDAI-2K), the Safety of Oestrogen in Lupus Erythematosus National Assessment (SELENA)-SLEDAl and the Physician Global Assessment (PGA, 0-3 scale) in each visit. Low-activity SLE (LLDAS) was defined according to Franklyn et al: 1) a SLEDAI-2K score $\leq 4,2$ ) no new SLE-related activity events compared with the previous visit, 3) a PGA score $\leq 1,4$ ) prednisone dose $\leq 7.5 \mathrm{mg}$ daily and 5) stable doses of maintenance immunosuppressant therapy. LLDAS was evaluated at baseline and months 3 and 6 . In order to adjust doses to weight, and given the impossibility of fractionating $\mathrm{HCQ}$ tablets, the adjusted dose was calculated for every week. For example, for $62 \mathrm{~kg}$ patient, the calculated dose would be 5 $\mathrm{mg} \times 52 \mathrm{~kg}=260 \mathrm{mg}$ daily, which would mean $1820 \mathrm{mg}$ weekly. Therefore, the patient would receive $400 \mathrm{mg}$ daily for 2 days and $200 \mathrm{mg}$ daily for 5 days every week. A relapse was defined as an increase of SLEDAI scores $\geq 3$, according to current recommendations

Results: A total of 50 LLDAS patients were compared, 25 with a fixed dose and 25 with a weight-adjusted dose. No significant differences regarding body weight between groups were observed: $52 \mathrm{~kg}(41-58.5 \mathrm{~kg})$ vs. $51 \mathrm{~kg}(44-55 \mathrm{~kg})$. Baseline activity scale scores were not significantly different. No patient relapsed on follow-up in either of both groups. Finally, the mean HCQ cumulative dose at 6 months was reduced by approximately 9.6 grams.

Conclusions: In LLADS patients, a fixed HCQ dose of $200 \mathrm{mg}$ daily may be as effective as the weight-adjusted dose in preventing disease relapses, thus resulting in a decrease in the HCQ cumulative dose and, therefore, a reduction of the risk of ocular toxicity.

\section{REFERENCE:}

[1] Franklyn K, Lau CS, Navarra SV, et al. Definition and initial validation of a Lupus Low Disease Activity State (LLDAS). Ann Rheum Dis 2016;75:1615-21.

Disclosure of Interest: None declared

DOI: 10.1136/annrheumdis-2018-eular.7414

\section{AB0509 KZR-616, A SELECTIVE INHIBITOR OF THE IMMUNOPROTEASOME, SHOWS A PROMISING SAFETY AND TARGET INHIBITION PROFILE IN A PHASE I, DOUBLE-BLIND, SINGLE (SAD) AND MULTIPLE ASCENDING DOSE (MAD) STUDY IN HEALTHY VOLUNTEERS}

J. Lickliter ${ }^{1}$, D. Bomba ${ }^{2}$, J. Anderl ${ }^{2}$, A. Fan ${ }^{2}$, C.J. Kirk ${ }^{2}$, J. Wang ${ }^{2} .{ }^{1}$ Nucleus Network, Melbourne, Victoria, Australia; ${ }^{2}$ Kezar Life Sciences, South San Francisco, California, USA

Background: Proteasome inhibition is a standard of care for plasma cell malignancies. Bortezomib targets the constitutive proteasome and immunoproteasome, and is effective in the treatment of Systemic Lupus Erythematosus (SLE) and lupus nephritis (LN), but is associated with hematologic (e.g. thrombocytopenia) and constitutional (e.g. peripheral neuropathy) adverse events (AEs).

Objectives: We report the safety, pharmacokinetics (PK), pharmacodynamics (PD) and immunomodulatory effects of KZR-616, a first-in-class selective inhibitor of the immunoproteasome, in healthy volunteers (HV) following single and repeat subcutaneous (SC) administration.

Methods: Cohorts (6 drug:2 placebo) received single or 4 weekly SC doses. Safety assessments, PK and PD were measured to Day 7 (SAD) or Day 28 (MAD). SAD cohorts included 7.5, 15, 30 and $60 \mathrm{mg}$ (SC). MAD cohorts included $30,45,60 \mathrm{mg}$, and 2 intrasubject escalation cohorts with 1 dose at $30 \mathrm{mg}$ and 3 doses at $45 \mathrm{mg}$. PK was measured by LC/MS ${ }^{2}$. PD was measured using enzymatic and active site binding assays. Inflammatory cytokine release was measured following ex vivo stimulation of whole blood in HV receiving placebo or $45 \mathrm{mg} \mathrm{KZR-616.}$

Results: 32 HV (24:8) were enrolled in 4 SAD cohorts. The most common AEs were injection site reactions (ISRs), which were generally mild and transient. No clinically-significant (CS) laboratory or ECG abnormalities and no dose limiting toxicities were observed in the SAD subjects. Following SC administration, drug exposure increased dose proportionally and was characterised by rapid absorption ( $T_{\max } 15-30 \mathrm{~min}$ ) and clearance ( $T_{1 / 2} \sim 2$ hours). Inhibition of the immunoproteasome exceeded $80 \%$ at $\geq 30 \mathrm{mg}$ with significant recovery noted over 7 days. Constitutive proteasome inhibition was $<37 \%$ in all cohorts.

$40 \mathrm{HV}$ (30:10) were enrolled in 5 SC MAD cohorts. In the initial cohort of $60 \mathrm{mg}$, a systemic drug reaction (chills, elevated heart rate, nausea) occurred $\sim 8$ hours after the first dose in 4 subjects. Dosing for this cohort was stopped. Subsequent cohorts (initiated at $30 \mathrm{mg}$ ) received prophylactic treatment with antihistamines \pm prednisone 1 hour prior to the first and second dose. No similar AEs occurred with repeat dosing of $45 \mathrm{mg}$. No CS laboratory or ECG abnormalities were seen in the remaining MAD cohorts. ISRs did not appear to increase in severity or frequency with repeat dosing. There were no AEs of peripheral neuropathy, and $45 \mathrm{mg}$ was well tolerated across 3 cohorts.

Consistent PK was noted following repeat administration and no drug accumulation was observed. At $45 \mathrm{mg}$, inhibition of 2 key subunits of the immunoproteasome, LMP7 and LMP2, was $\sim 95 \%$ and $70 \%$, respectively. Reduced ex vivo stimulated production of multiple cytokines (IL-23, IL-6, TNF-a, IL-17) was noted in subjects receiving repeat administration of $45 \mathrm{mg} \mathrm{KZR-616.}$ 